\title{
Phenotype, Virulence and Immunogenicity of Edwardsiella piscicida Cyclic AMP Receptor Protein (Crp) Mutants in Catfish Host
}

\author{
Peng Zhou ${ }^{1,+}$, Xueqing Han ${ }^{1,+}{ }^{,}$Xiang Ye ${ }^{1}$, Feifei Zheng ${ }^{1}$, Ting Yan ${ }^{2}$, Quan Xie ${ }^{1}$, \\ Yong-An Zhang ${ }^{1}{ }^{\circledR}$, Roy Curtiss III ${ }^{3, *}$ and Yang Zhou ${ }^{1, *}$ \\ 1 Department of Aquatic Animal Medicine, College of Fisheries, Huazhong Agricultural University, \\ Wuhan 430070, China; 15926316870@163.com (P.Z.); xqhan94@163.com (X.H.); 18086042051@163.com (X.Y.); \\ zhengfeifei@mail.hzau.edu.cn (F.Z.); 635412738@webmail.hzau.edu.cn (Q.X.); \\ yonganzhang@mail.hzau.edu.cn (Y.-A.Z.) \\ 2 College of Food Science and Technology, Huazhong Agricultural University, Wuhan 430070, China; \\ yt15827390282@163.com \\ 3 Department of Infectious Disease and Immunology, College of Veterinary Medicine, University of Florida, \\ Gainesville, FL 32608, USA \\ * Correspondence: zhouyang@mail.hzau.edu.cn (Y.Z.); rcurtiss@ufl.edu (R.C.I.); Tel.: +86-27-87282113 (Y.Z.); \\ +352-294-4086 (R.C.I.); Fax: +86-27-87282114 (Y.Z.); +352-392-9704 (R.C.I.) \\ + These authors contributed equally to this work.
}

Received: 1 March 2020; Accepted: 2 April 2020; Published: 4 April 2020 updates

\begin{abstract}
Edwardsiella piscicida, a facultative aerobic pathogen belonging to the Enterobacteriaceae family, is the etiological agent of edwardsiellosis that causes significant economic loses in the aquaculture industry. cAMP receptor protein (CRP) is one of the most important transcriptional regulators, which can regulate large quantities of operons in different bacteria. Here we characterize the crp gene and report the effect of a crp deletion in E. piscicida. The crp-deficient mutant lost the capacity to utilize maltose, and showed significantly reduced motility due to the lack of flagella synthesis. We further constructed a $\Delta \mathrm{P}_{\text {crp }}$ mutant to support that the phenotype above was caused by the $c r p$ deletion. Evidence obtained in fish serum killing assay and competitive infection assay strongly indicated that the inactivation of crp impaired the ability of E. piscicida to evade host immune clearance. More importantly, the virulence of the crp mutant was attenuated in both zebrafish and channel catfish, with reductions in mortality rates. In the end, we found that $\operatorname{crp}$ mutant could confer immune protection against E. piscicida infection to zebrafish and channel catfish, indicating its potential as a live attenuated vaccine.
\end{abstract}

Keywords: Edwardsiella piscicida; cAMP receptor protein; virulence; vaccine

\section{Introduction}

Edwardsiella piscicida is a facultative anaerobe and Gram-negative enteric pathogen that generally causes lethal edwardsiellosis, which is a systematic enterohemorrhagic septicemic disease that can lead to high stock mortality in fresh and marine fish [1,2]. E. piscicida infection induces symptoms including emphysematous putrefactive disease with swelling skin lesions, as well as gill ulceration and necrosis in internal organs such as kidney, liver, spleen, and musculature [2,3].

To date, antimicrobial susceptibility testing shows that E. piscicida strains isolated from different hosts and geographical regions are susceptible to most commonly used antibiotics for the treatment of edwardsiellosis [4]. However antibiotic abuse represents a problematic method of treating bacterial infections in the aquaculture industry and has led to the evolution of multi-drug resistance strains [5]. 
Plasmid-mediated multi-drug resistant has been previously reported in E. piscicida [6]. In response to this potential, vaccination is an important disease control strategy that has significantly contributed to reduction of outbreaks and antibiotics use in aquaculture [7]. It is worth noting that intracellular bacterial vaccines evoke cellular mediated immune responses that "kill" and eliminate infected cells [7]. The intracellular lifestyle of E. piscicida has been gradually revealed. This pathogen could successfully survive and replicate within fish phagocytes [8]. In summary, the above attributes make E. piscicida a promising candidate to be developed as a live attenuated vaccine against edwardsiellosis.

The cyclic AMP receptor protein (Crp), also called catabolite gene activator protein (CAP), is member of the CRP-FNR (fumarate nitrate reductase regulator) superfamily of transcription factors [9]. In the lack of a preferred carbon source (e.g., glucose), the Crp-cAMP complex contributes to the phenomenon of catabolite repression, inducing catabolic pathways for growth on alternative substrates [10]. Crp enhances the ability of the RNA polymerase holoenzyme to bind and initiate the transcription of specific sets of genes. In Escherichia coli, there are currently estimated to be more than 100 operons and $\sim 500$ genes under the control of Crp-cAMP [11]. The Crp-cAMP complex also contributes to the regulation of virulence gene expression in many pathogenic bacteria including Salmonella, Vibrio cholerae, Yersinia, and Mycobacterium tuberculosis [12-15]. crp deletion mutants have been successfully developed as live attenuated bacterial vaccines to protect various animal species against different bacterial pathogens [12,16-18]. In fish pathogens, mutation of $c r p$ in Aeromonas salmonicida attenuated its virulence as $\sim 6$ times in Oncorhynchus mykiss. And the $\Delta$ crp mutant could confer protective immunity against the intraperitoneal challenge with $A$. salmonicida wild type [19]. And in E. ictaluri, crp mutant was also attenuated and conferred immune protection against E. ictaluri challenge to zebrafish (Danio rerio) and catfish (Ictalurus punctatus) [20]. In this study, we investigated the effects of a crp gene deletion on E. piscicida physiology, virulence and ability to confer immune protection to fish hosts.

\section{Materials and Methods}

\subsection{Ethics Statement}

All animal experimental work was approved by the Laboratory Animal Monitoring Committee of Huazhong Agricultural University. Procedures conducted involving in animals were in accordance with the suggestions of the Guide for the Care and Use of Laboratory Animals of Hubei Province, China.

\subsection{Bacterial Strains and Growth Conditions}

The bacterial strains used in the study are listed in Table 1. E. piscicida was routinely cultured in Luria broth (LB) or on Luria agar (LA) plates (Difco, Detroit, MI, USA) at $28^{\circ} \mathrm{C}$. When necessary, $0.2 \%$ arabinose was supplemented to activate the $\mathrm{P}_{\mathrm{BAD}}$. Escherichia coli $\chi 7213$ was used for mutant plasmid harvest and was cultured in LB broth at $37^{\circ} \mathrm{C}$, supplemented with diaminopimelic acid (50 g/L) (Sigma, St. Louis, MO, USA). When necessary, ampicillin (Sigma, St. Louis, MO, USA) and chloramphenicol (Sigma, St. Louis, MO, USA) were supplemented at final concentrations of 100 and $50 \mu \mathrm{g} / \mathrm{mL}$, respectively.

\subsection{Sequence Analysis}

The crp nucleic acid and Crp amino acid sequences from various bacteria species were downloaded from NCBI. The accession numbers were provided in the supplementary data. MAFFT 7 and ESPript 3 were used for multiple sequence alignment. The neighbor-joining method in Molecular Evolutionary Genetics Analysis package (MEGA 7.0) was used to construct a phylogenetic tree. The evolutionary distances were computed using the p-distance model. The E. piscicida Crp 3D structure was predicted using the Iterative Threading ASSEmbly Refinement (I-TASSER) server [21]. 


\subsection{Construction and Characterization of Crp Mutants}

Primers and plasmids used in this experiment are listed in Tables 1 and 2. The procedures for mutant construction were described previously [22]. The Primers P1/P2 and P3/P4 were used in amplifications of upstream or downstream flanking fragments of $c r p$ via PCR respectively. Then, the upstream and downstream homologous arms were fused by overlap PCR using primers P1/P4, and ligated into the suicide plasmid pRE112 at the XbaI/SacI sites. The resulting plasmid pRE112-crp was transformed into E. coli $\chi 7213$ for mobilization into E. piscicida via conjugation. Strains containing single-crossover plasmid insertions were isolated on LA media containing chloramphenicol. Loss of the suicide vector after the second recombination between homologous regions was selected by using the sacB-based sucrose-sensitivity counter-selection system. Resulting strain was selected on LA plates containing chloramphenicol. PCR identification via primers P1/P4 and direct DNA sequencing of the mutation sites using genomic DNA preparations were conducted to verify that the construction of the crp gene deletion strain was completed.

Table 1. Strains and plasmids used in this study.

\begin{tabular}{|c|c|c|}
\hline & Description & Source or reference \\
\hline \multicolumn{3}{|c|}{ Strains } \\
\hline \multicolumn{3}{|c|}{ Edwardsiella piscicida } \\
\hline $\mathrm{J} 118$ & pEIB202 curing derivative of EIB202, $\mathrm{Col}^{\mathrm{r}}, \mathrm{Cm}^{\mathrm{s}}, \mathrm{pla}^{-}$ & Lab stocking \\
\hline$\Delta c r p$ & crp deletion mutant & $\mathrm{J} 118$ \\
\hline$\Delta \mathrm{P}_{\mathrm{crp}}$ & $\Delta \mathrm{P}_{\mathrm{crp}} \mathrm{TT}$ araC $\mathrm{P}_{\mathrm{BAD}}$ crp $\mathrm{TT}$ & $\mathrm{J} 118$ \\
\hline \multicolumn{3}{|l|}{ Escherichia coli } \\
\hline$\chi 7213$ & thr-1 leuB6 fhuA21 lac $\mathrm{Y} 1$ glnV44 recA1 $\Delta a s d \mathrm{~A} 4 \Delta(z h f-2:: T n 10)$ thi-1 & [16] \\
\hline \multicolumn{3}{|l|}{ Plasmids } \\
\hline pYA3700 & $\mathrm{TT}$ araC $\mathrm{P}_{\mathrm{BAD}}$ cassette plasmid; $\mathrm{Ap}^{\mathrm{r}}$ & [23] \\
\hline pRE112 & Suicide vector, $\mathrm{sacB}, \mathrm{mob}^{-}(\mathrm{RP} 4) \mathrm{R} 6 \mathrm{~K}$ ori, $\mathrm{Cm}^{\mathrm{r}}$ & {$[23]$} \\
\hline pRE112-crp & pRE112 derivative, designed for knockout of $c r p, \mathrm{Cm}^{\mathrm{r}}$ & pRE112 \\
\hline
\end{tabular}

$\mathrm{Col}^{\mathrm{r}}$ stands for Colistin-resistant. $\mathrm{Cm}^{\mathrm{r}}$ stands for Chloramphenicol-resistant. Ap ${ }^{\mathrm{r}}$ stands for Ampicillin-resistant.

Table 2. Primers used in this study.

\begin{tabular}{|c|c|c|c|c|c|}
\hline Primer & Sequence $\left(5^{\prime}-3^{\prime}\right)$ & $\begin{array}{l}\text { Product } \\
\text { Size (bp) }\end{array}$ & Tm & Target Gene & $\begin{array}{l}\text { Source or } \\
\text { Reference }\end{array}$ \\
\hline P1 & CGCTCTAGACCACAGGACAAACCAAAACC & \multirow[t]{2}{*}{593} & \multirow[t]{2}{*}{58} & Upstream fragment for $\Delta c r p$ & \multirow[t]{2}{*}{ This study } \\
\hline $\mathrm{P} 2$ & TGCTGGAGGATCAGAACCTGATCTCGGCACACGGTAAAAC & & & construction & \\
\hline P4 & CCCGAGCTCAGAGACGCTGGATAGCCTGA & 579 & 58 & construction & This study \\
\hline P5 & CCCAGATCTTCTATACCCGCTTCATTCCA & \multirow[t]{2}{*}{557} & \multirow[t]{2}{*}{58} & Upstream fragment for $\Delta \mathrm{P}_{\text {crp }}$ & \multirow[t]{2}{*}{ This study } \\
\hline P6 & CGCAAGCTTCCCCGGGCCGTCCAATATCGAATACCA & & & construction & \\
\hline
\end{tabular}

We further constructed $\Delta \mathrm{P}_{\text {crp }}$ by replacing the promoter of crp gene with the arabinose-regulated araC $\mathrm{P}_{\mathrm{BAD}}$ promoter. The procedures were described previously [23]. A 557-bp DNA fragment containing the region upstream of the crp promoter was PCR amplified as a template with primers P5/P6 (Table 2). The PCR-amplified fragment was digested with BglII/HindIII and cloned into the vector pYA3700 (Table 1). A 595-bp PCR fragment, including 583-bp of crp gene and its original Shine-Dalgarno sequence was amplified using primers P7/P8 (Table 2). The PCR fragment was digested with $\mathrm{XhoI} / \mathrm{KpnI}$ and inserted into the intermediate plasmid described above. The resulting construct was confirmed by DNA sequence analysis. Then, a 2.5-kb DNA fragment including araC $\mathrm{P}_{\mathrm{BAD}}, \mathrm{P}_{\mathrm{crp}} 5^{\prime}$ and $3^{\prime}$ flanking regions were excised from the plasmid by digestion with $\mathrm{KpnI} / \mathrm{XmaI}$ and inserted into pRE112, resulting in plasmid pRE112- $\Delta \mathrm{P}_{\text {crp }}$. The following procedure was the same as that in $\Delta c r p$ mutant construction.

To determine the growth kinetics of different strains, 1:100 diluted overnight cultures were cultured in LB medium at $28^{\circ} \mathrm{C}$. Samples were taken hourly, and the optical densities were measured at $600 \mathrm{~nm}$ 
$\left(\mathrm{OD}_{600 \mathrm{~nm}}\right)$. In addition, on $2 \mathrm{~h}, 4 \mathrm{~h}, 6 \mathrm{~h}, 8 \mathrm{~h}, 10 \mathrm{~h}, 12 \mathrm{~h}, 24 \mathrm{~h}, 36 \mathrm{~h}$ post inoculation, the numbers of $\mathrm{CFU}$ in cultures were determined by serial dilutions and plating.

In order to study whether CRP is involved in the process of maltose utilization, the growth of $\mathrm{crp}$ mutant strains and parent strain were observed on MacConkey agar supplemented with maltose (1\%). Swimming motility was measured on LA plates that were prepared with $0.3 \%(w / v)$ agar according to the method described previously [20]. To increase flagella synthesis, the bacterial samples were collected from motility agar plates. Negative staining method was used to observe the bacteria morphology under transmission electron microscopy (TEM; Hitachi H-7000FA, Tokyo, Japan). Ten microliters of the resuspension of each strain was applied to Formvar-coated copper grids and negatively stained for 1 min with $1 \%$ uranyl acetate [20].

\subsection{Resistance Against Host Clearance of E. piscicida}

To investigate resistance of the parent J118 strain and $\Delta c r p$ against serum killing, a catfish serum survival assays was accomplished as previously described [24]. Blood was withdrawn from the caudal vein of channel catfish (average weight, $500 \mathrm{~g}$ ) by sterile injector and placed on ice immediately. The blood was allowed for clotting over night at $4{ }^{\circ} \mathrm{C}$. For serum survival assay, the serum was treated with or without heating at $56^{\circ} \mathrm{C}$ for $1 \mathrm{~h}$. A mid-log growth phase inoculum of $5.0 \times 10^{5} \mathrm{CFU}$ bacteria in $100 \mu \mathrm{L}$ was mixed with $400 \mu \mathrm{L}$ serum. The mixtures were incubated at $28^{\circ} \mathrm{C}$ for $1 \mathrm{~h}$. Then, the numbers of CFU were determined by serial dilutions and plating. The survival percentage was subsequently calculated as follows: (CFUs after co-incubation /CFUs in PBS control group) $\times 100 \%$. E. coli $\mathrm{DH} 5 \alpha$ strain was used as a sensitive control strain in this assay. The experiments were repeated three times independently.

To determine the persistent carrier state of J118 and $\Delta c r p$ strains in host, the competitive assay was performed as previously described [22]. J118 and $\Delta c r p$ strains grown to mid-exponential phase in LB were harvested by centrifugation, washed and resuspended in PBS to a bacterial density of $5.0 \times 10^{6} \mathrm{CFU} / \mathrm{mL}$, respectively. In three independent assays, a total of eleven channel catfish were injected by the intracoelomic (i.c.) route with $200 \mu \mathrm{L}$ volume of 1:1 $\Delta$ crp mutant - J118 mixture $\left(1.0 \times 10^{6} \mathrm{CFU} /\right.$ fish). Twenty-four hours later, fish were euthanized and the blood, ascites, liver, spleen, trunk kidney and head kidney samples were collected. Homogenates were serially diluted and plated on MacConkey agar with maltose $(1 \%)$ at $37^{\circ} \mathrm{C}$ for $24 \mathrm{~h}$. The parent strain is red and the $\Delta c r p$ mutant is white on the plate, respectively. Bacterial counts titers were calculated by dividing the weights of the tissues from the bacteria loads in the samples.

\subsection{Virulence of E. piscicida $\Delta$ crp in Zebrafish and Catfish Fingerlings}

The zebrafish (average weight, $300 \mathrm{mg}$ ) used in this study were from the Institute of Hydrobiology, Chinese Academy of Sciences (Wuhan, China). The zebrafish were acclimatized for 2 weeks before injection. They were fed with commercial feed for aquatic animal twice per day under natural photoperiod. The water temperature was maintained at $24-26{ }^{\circ} \mathrm{C}$ during cultivation. Zebrafish are divided into 12 groups, each group of 10, infected with J118 strain and $\Delta c r p$ respectively (doses: $10^{2} \mathrm{CFU} /$ fish, $10^{3} \mathrm{CFU} /$ fish, $10^{4} \mathrm{CFU} /$ fish, $10^{5} \mathrm{CFU} /$ fish, $10^{6} \mathrm{CFU} /$ fish, and $10^{7} \mathrm{CFU} /$ fish). Chanel catfish (average weight, $800 \mathrm{mg}$ ) from a local aquaculture farm were acclimatized and monitored for 2 weeks before infection. Catfish were fed daily with commercially produced food pellets (Haida, China) under natural photoperiod. Water temperature was maintained at $23-25^{\circ} \mathrm{C}$. Catfish are divided into 10 groups, each group of 10, i.c. infected with J118 strain and the $\Delta c r p$ mutant, respectively (doses: $10^{4} \mathrm{CFU} /$ fish, $10^{5} \mathrm{CFU} /$ fish, $10^{6} \mathrm{CFU} /$ fish, $10^{7} \mathrm{CFU} /$ fish, and $10^{8} \mathrm{CFU} /$ fish). The clinical symptoms and mortality of infected fish were observed for 14 days. The $\mathrm{LD}_{50}$ was calculated by Karber's methods [25]. 


\subsection{Immune Protection of E. piscicida $\Delta c r p$ Mutant in Zebrafish and Catfish Fingerlings}

We further evaluated the immune protection mediated by $\Delta c r p$ in zebrafish and catfish fingerlings. Fish were divided into immunized group and control group, with 10 zebrafish in each group and 20 catfish fingerling in each group. Zebrafish and catfish were i.c. immunized with $10^{3} \mathrm{CFU} /$ fish and $10^{4} \mathrm{CFU} /$ fish of $\Delta c r p$, respectively. Subsequent booster immunization was given at day 14 post-primary immunization. At 2 weeks post-booster immunization, zebrafish and catfish were challenged i.c. with 10-times $\mathrm{LD}_{50}$ E. piscicida J118. The fish were observed for clinical symptoms and mortalities for 14 days.

\subsection{Statistical analysis}

Statistical analysis was performed by GraphPad Prism 6 (Graph Pad Software, Inc., San Diego, CA, USA). Survival data were analyzed with the log-rank (Mantel-Cox) test. The statistical p values were calculated by the two-tailed Mann-Whitney $\mathrm{t}$ test. Differences were considered significant at $p<0.05$ and highly significant at $p<0.01$.

\section{Results}

\subsection{Sequence Analysis}

The catabolic and virulence regulator crp gene is wide spread not only between enteric pathogens, including E. piscicida, but also within other bacterial domains (Figure 1). The phylogenetic tree clearly showed crp genes in Enterobacteriaceae, Aeromonadaceae, Vibrionaceae and Pasteurellaceae, belonging to $\gamma$-Proteobacteria, were clustered in clade A. The closely related clade B includes other Proteobacteria, while the clade $\mathrm{C}$ includes Cyanobacteria, Actinobacteria, Bacteroidetes and Firmicutes, with a more distantly evolutionary relationship. Especially, crp genes in enteric fish pathogens including two Aeromonas species and two Edwardsiella species showed close genetic relationship according to this phylogenetic tree.

Sequence and structural alignment between functional representative bacterial Crp proteins revealed that 189 amino acid residues (90\%) are strictly conserved out of 210 residues in E. piscicida Crp (Figure 2A). E. piscicida Crp has 100.0\%, 99.0\%, 98.6\%, and 89.0\% amino acid similarity to the Crp of Escherichia coli, E. ictaluri, Yersinia pestis and Aeromonas hydrophila, respectively. The conserved cAMP binding amino acid residues: Gly72, Glu73, Arg83, Ser84, Thr128, and Ser129 (Figure 2A) of E. piscicida Crp is similar to other Gram-negative Crp-family members. The E. piscicida Crp 3-D predicted structure exhibits the flexible hinge required for Crp dimerization and the F-helix \& C-helix that interacts with the DNA (Figure 2B). Thus, above results shows that Crp is conserved and indicates its function is conserved through the evolution of the Enterobacteriaceae family, including enteric fish pathogens.

\subsection{Construction and Characterization of Crp Mutants}

To investigate the role of Crp in the pathogenesis of E. piscicida, a crp deletion mutant was constructed by allelic replacement in parent strain J118 (Figure 3A). The growth of E. piscicida strains was quantified over time by measuring the $\mathrm{OD}_{600 \mathrm{~nm}}$ and CFUs in cultures (Figure 3B). Although the $\mathrm{OD}_{600 \mathrm{~nm}}$ growth curve showed growth defect of $\Delta c r p$ in LB broth, the CFUs showed no differences between $\Delta c r p$ mutant strain and parent strain, which indicated that the mutation of crp affected the secretion of bacterial extracellular products. The phenotype by growth on MacConkey agar supplemented with maltose $(1 \%)$ shows that the $\Delta c r p$ mutant does not utilize maltose, indicating that the genes related to maltose utilization is under the positive regulation by Crp (Figure 4A). Except for involvement in catabolic functions, the Crp regulatory complex is also necessary for the flagella synthesis. We evaluated the motility of the $\Delta$ crp mutant in LB broth supplemented with $0.3 \%$ agar and flagella synthesis by TEM. The results showed that the $\triangle c r p$ mutant loses motility (Figure $4 \mathrm{~B}$ ) due to the lack of flagella synthesis (Figure 4C), indicating that Crp positively regulates flagella synthesis in E. piscicida. 


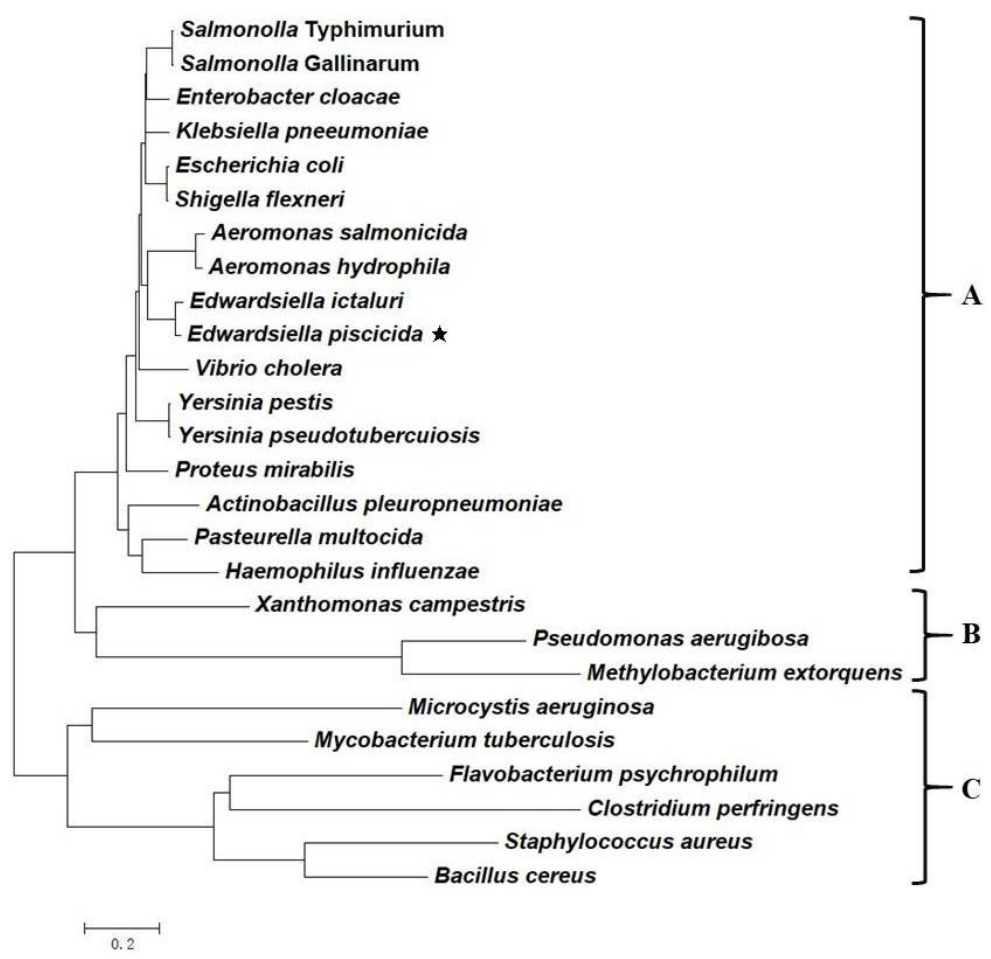

Figure 1. Phylogenetic tree of crp gene. The evolutionary history was inferred using Molecular Evolutionary Genetics Analysis (MEGA) 7.0 with neighbor-joining method. The tree is drawn to scale, with branch lengths in the same units as those of the evolutionary distances used to infer the phylogenetic tree. The analysis involved 26 nucleotide sequences. E. piscicida crp was marked with $\star$.

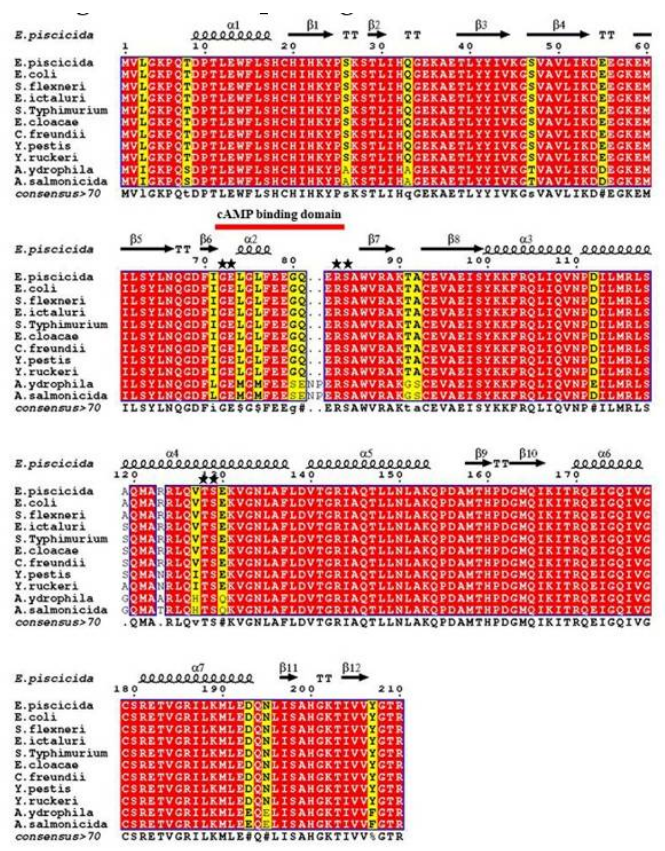

B

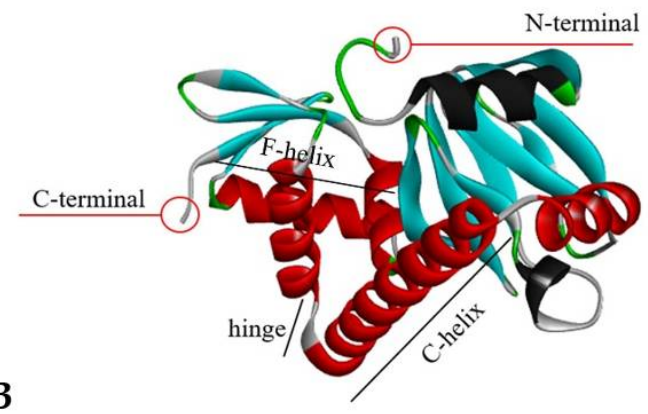

Figure 2. Multiple alignments of Crp and 3-D predicted structure. (A) Secondary structure of E. piscicida Crp and alignment between representative Crp proteins. The secondary structure at the top of the alignment corresponds to the E. piscicida Crp (spirals represent $\alpha$-helix; arrows represent $\beta$-sheet). Conserved amino acids residues are indicated in red. The stars indicate the amino acid residues required for cAMP binding. (B) E. piscicida Crp monomer structure. The helixes required for DNA binding and dimer Crp formation are indicated (C-helix, F-helix, and hinge). The N- and C-termini are indicated in a red circle. 
A
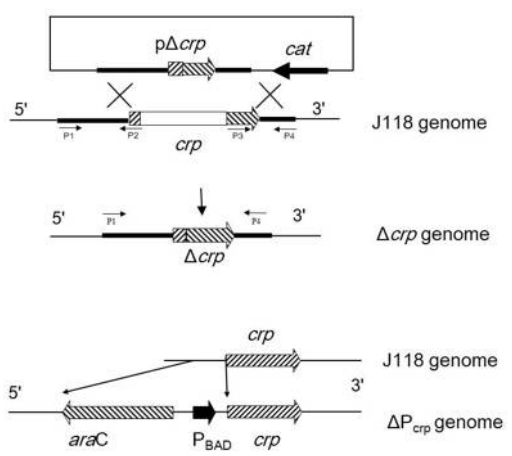

B
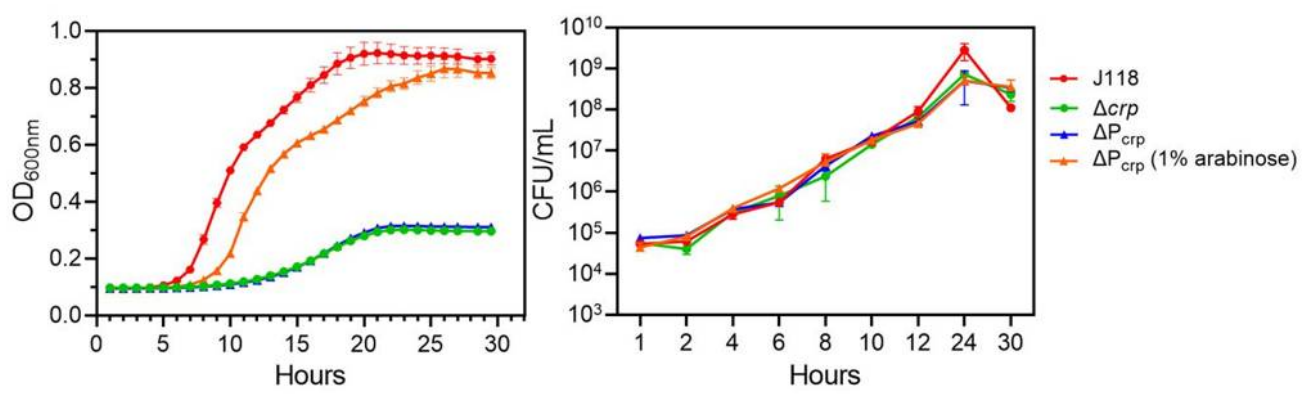

Figure 3. Construction and growth curves of crp mutants. (A) Strategy for construction of $\Delta c r p$ and $\Delta \mathrm{P}_{\text {crp }}$ by homologous recombination. (B) Growth curves in LB medium over a $30 \mathrm{~h}$ period. Data are presented as the averages \pm the standard deviations for three replicates.

A

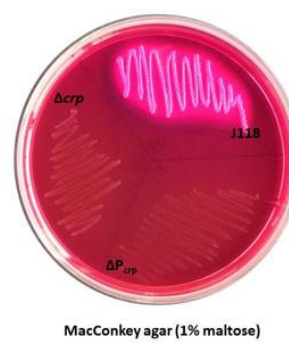

MacConkey agar $(1 \%$ maltose $)$

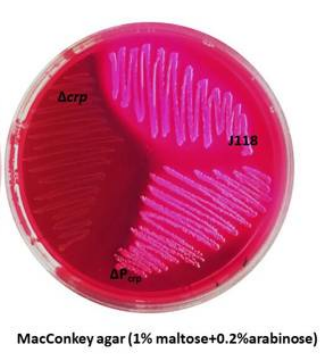

MacConkey agar ( $1 \%$ maltose+0. $2 \%$ arabinose $)$
B

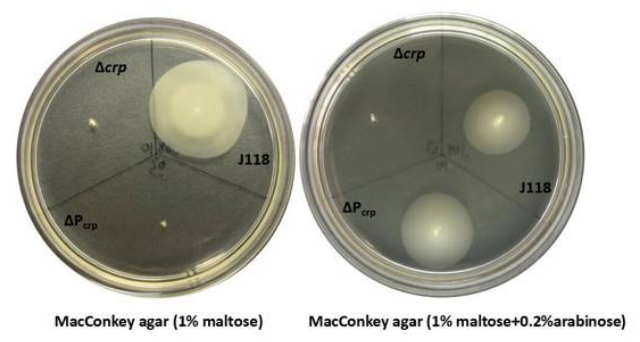

C

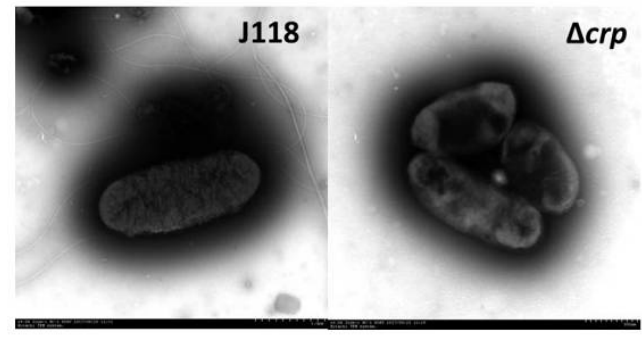

Figure 4. Phenotype characterization of crp mutants. (A) Phenotype verification on MacConkey agar plates supplemented with $1 \%$ maltose and with or without $0.2 \%$ arabinose. (B) Swimming zones through $0.3 \%$ LB agar with or without $0.2 \%$ arabinose. (C) Negative staining-transmission electron microscopy of E. piscicida parent strain J118 (scale bar $=1 \mu \mathrm{m}$ ) and crp mutant (scale bar $=500 \mathrm{~nm}$ ).

We further constructed a $\Delta \mathrm{P}_{\text {crp }}$ mutant by replacing the promoter of crp gene with the arabinose-regulated ara $\mathrm{C}_{\mathrm{BAD}}$ promoter (Figure 3A). The transcription of $\mathrm{crp}$ is dependent upon arabinose availability. The $\Delta \mathrm{P}_{\text {crp }}$ showed similar phenotypes with $\Delta c r p$ mutant in the absence of 
arabinose (Figure 3B, Figure 4A,B). However, when supplemented with arabinose, the defects in growth, maltose utilization and motility were all well complemented (Figure 3B, Figure 4A,B). Above results verify that crp mutation, instead of other random mutation, contributes to the phenotypes differences.

\subsection{Resistance Against Host Clearance of E. piscicida $\Delta$ crp}

To investigate the role of crp in E. piscicida resistance against innate immune responses, we first examined the abilities of the strains to survive in a channel catfish serum survival assay. When E. piscicida J118 strain was incubated with catfish serum, the survival rate of the bacteria was found to be $104.3 \%$, revealing that parent strain resisted the bactericidal effect of catfish serum. $\Delta c r p$ mutant had showed a significantly reduced survival rate (40.0\%) compared with the parent strain (Figure $5 \mathrm{~A})$. The survival rate of $E$. coli $\mathrm{DH} 5 \alpha$, a serum-sensitive laboratory strain incubated under the same condition, was $0 \%$. When incubated with inactivated serum, the $\Delta c r p$ mutant showed an increased survival rate of $71.6 \%$, but still lower than parent strain. Above results suggested that the crp mutant could poorly resist the killing by bactericidal substances in fish serum, and then could not effectively evade the host clearance.

A

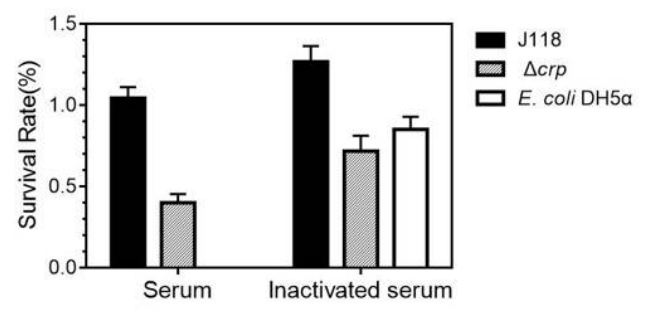

B
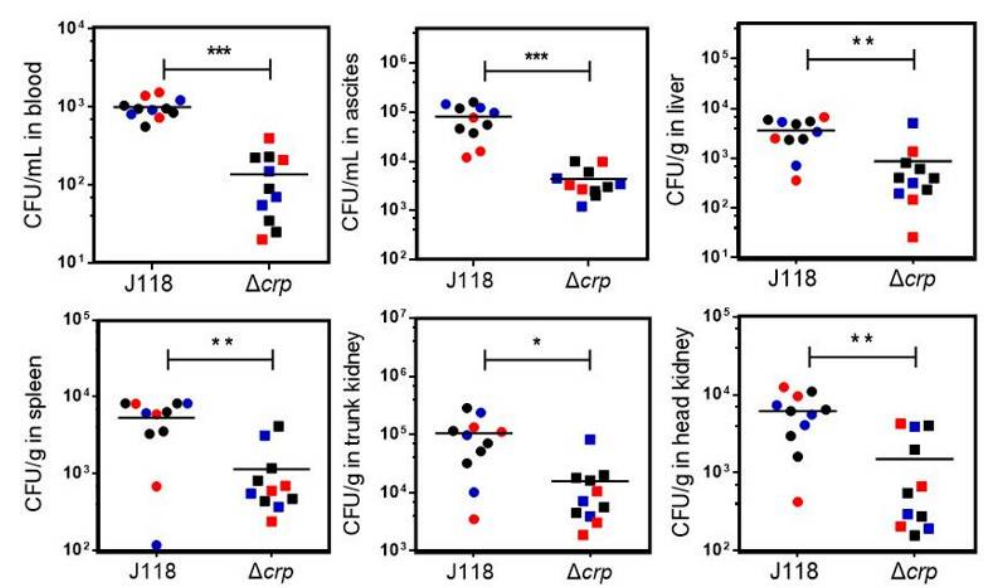

Figure 5. Effect of Crp deletion on resistance against host clearance. (A) Percentage of CFUs following $1 \mathrm{~h}$ of incubation with catfish serum, or heat-inactivated catfish serum. (B) Colonization of channel catfish tissues by E. piscicida J118 and $\Delta c r p$ mutant in competitive infection assay. The data was a combination the three independent assays. ${ }^{*} p<0.05,{ }^{* *} p<0.01,{ }^{* * *} p<0.001$.

To test this idea, we performed a competitive infection assay. An approximately 1:1 mixture of J118 and $\Delta c r p$ bacteria was inoculated by the intracoelomic (i.c.) route into channel catfish. Twenty-four hours later, blood, ascites, liver, spleen, trunk kidney and head kidney samples were collected after the fish were euthanized. Comparing to the parent strain, $\Delta c r p$ colonized at significantly lower levels in all the samples (Figure 5B), which confirmed that the crp mutation impaired the resistance ability of E. piscicida against innate immune clearance in vivo. 


\subsection{Virulence of E. piscicida $\Delta$ crp in Zebrafish and Catfish Fingerling}

The ideal live attenuated bacterial vaccine should be totally attenuated. Zebrafish have been developed as an easy and powerful model to test pathogenesis of E. piscicida. The $\mathrm{LD}_{50}$ of the parent strain J118 was $7.94 \times 10^{3} \mathrm{CFU}$, while the $\mathrm{LD}_{50}$ of the $\Delta c r p$ mutant increased up to $3.16 \times 10^{5} \mathrm{CFU}$, nearly 40-fold compared to the parental strain (Table 3). For the zebrafish infected with J118, the death occurred immediately during the first $24 \mathrm{~h}$, while it was quite delayed in the $\Delta$ crp infection group (Figure 6A). The E. piscicida $\Delta c r p$ mutant was further evaluated in the channel catfish host. We found that the $\Delta c r p$ mutant applied by the i.c. route was attenuated with an estimated 10 -times $\mathrm{LD}_{50}$ increase over the parent strain (Table 4). Catfish fingerlings that were infected with J118 developed the typical symptoms of enteric septicemia, skin lesions and distended abdomen, while the ones infected with the $\Delta c r p$ mutant exhibited mild symptoms (Figure 6B). Taken together, these results indicate that $\mathrm{Crp}$ significantly contributed to the virulence of E. piscicida.

Table 3. Calculations of $\mathrm{LD}_{50} \mathrm{~s}$ of $\mathrm{J} 118$ and $\Delta c r p$ mutant strains in zebrafish.

\begin{tabular}{|c|c|c|c|c|}
\hline \multirow{2}{*}{$\begin{array}{c}\text { Dose of Challenge } \\
\text { CFU }\end{array}$} & \multicolumn{2}{|c|}{ Number of Death/Total } & \multicolumn{2}{|c|}{ Survival Rate (\%) } \\
\hline & J118 & $\Delta c r p$ & J118 & $\Delta c r p$ \\
\hline $1.0 \times 10^{7}$ & - & $10 / 10$ & - & 0 \\
\hline $1.0 \times 10^{6}$ & $10 / 10$ & $6 / 10$ & 0 & 40 \\
\hline $1.0 \times 10^{5}$ & $8 / 10$ & $3 / 10$ & 20 & 70 \\
\hline $1.0 \times 10^{4}$ & $6 / 10$ & $1 / 10$ & 40 & 90 \\
\hline $1.0 \times 10^{3}$ & $2 / 10$ & $0 / 10$ & 80 & 100 \\
\hline $1.0 \times 10^{2}$ & $0 / 10$ & - & 100 & - \\
\hline $\mathrm{LD}_{50} *$ & $7.94 \times 10^{3}$ & $3.16 \times 10^{5}$ & & \\
\hline
\end{tabular}

* The $\mathrm{LD}_{50}$ was calculated according to Karber's method.

A

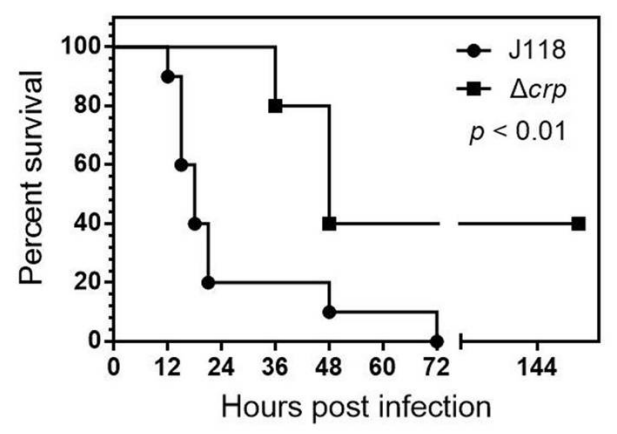

B

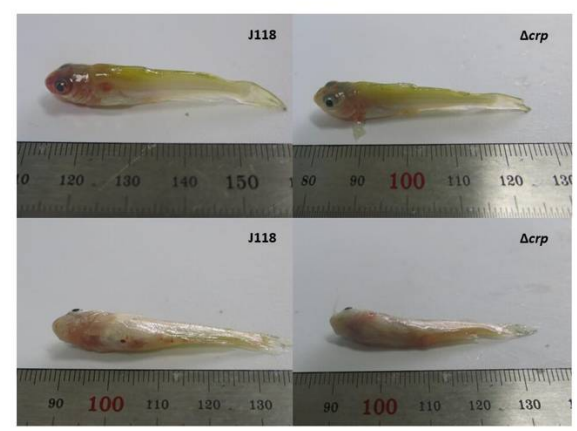

Figure 6. (A) Survival curves for zebrafish challenged with E. piscicida J118 and $\Delta$ crp mutant $\left(1.0 \times 10^{6} \mathrm{CFU}\right)$. (B) Catfish fingerlings i.c. infected with E. piscicida J118 and $\Delta$ crp mutant $\left(1.0 \times 10^{6} \mathrm{CFU}\right)$.

Table 4. Calculations of $\mathrm{LD}_{50} \mathrm{~s}$ of $\mathrm{J} 118$ and $\Delta c r p$ mutant strains in channel catfish.

\begin{tabular}{ccccc}
\hline Dose of Challenge & \multicolumn{2}{c}{ Number of Death/Total } & \multicolumn{2}{c}{ Survival Rate (\%) } \\
CFU & J118 & $\Delta$ crp & J118 & $\Delta$ crp \\
\hline $1.0 \times 10^{8}$ & - & $10 / 10$ & - & 0 \\
$1.0 \times 10^{7}$ & $10 / 10$ & $7 / 10$ & 0 & 30 \\
$1.0 \times 10^{6}$ & $5 / 10$ & $0 / 10$ & 50 & 100 \\
$1.0 \times 10^{5}$ & $2 / 10$ & - & 80 & - \\
$1.0 \times 10^{4}$ & $0 / 10$ & - & 100 & - \\
$\mathrm{LD}_{50}{ }^{*}$ & $6.31 \times 10^{5}$ & $6.31 \times 10^{6}$ & \\
\hline
\end{tabular}




\subsection{Immune Protection of E. piscicida Acrp Mutant in Zebrafish and Catfish Fingerling}

Previous results indicate that the E. piscicida $\Delta c r p$ mutant had reduced virulence and that the host is able to control the infection. We further evaluated the immune protection mediated by the $\Delta c r p$ mutant in zebrafish and catfish fingerlings. At 2 weeks post-booster immunization, zebrafish were challenged i.c. with 10-times $\mathrm{LD}_{50}$ E. piscicida J118. The $\Delta$ crp mutant applied by the i.c. route was immune protective in the zebrafish host, with $70 \%$ survival while only $10 \%$ survival in the PBS control group (Figure 7A). A challenge study was also performed to determine whether the $\Delta c r p$ mutant induces protective immunity in catfish fingerlings. At 2 weeks post-booster immunization, catfish fingerlings were challenged i.c. with 2-times $\mathrm{LD}_{50}$ E. piscicida J118. The $\Delta$ crp mutant showed a $90 \%$ survival rate against J118 infection, which is significantly higher than that of PBS (Figure 7B).

A

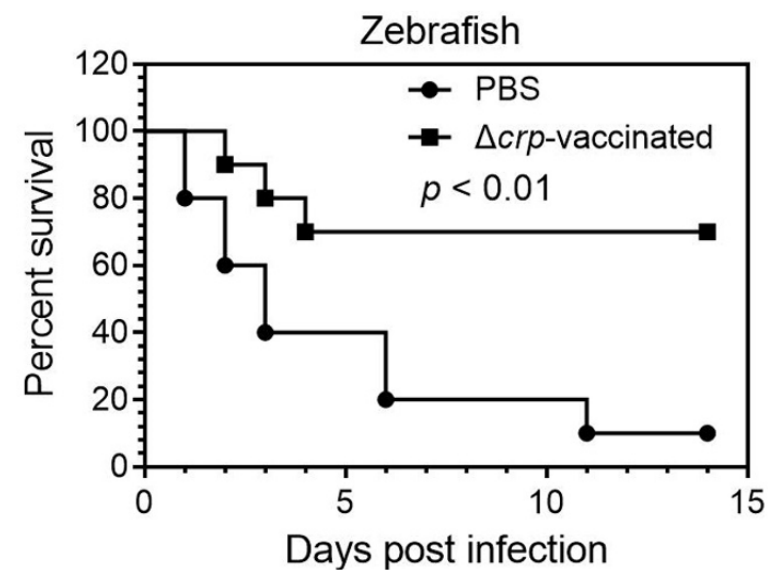

B

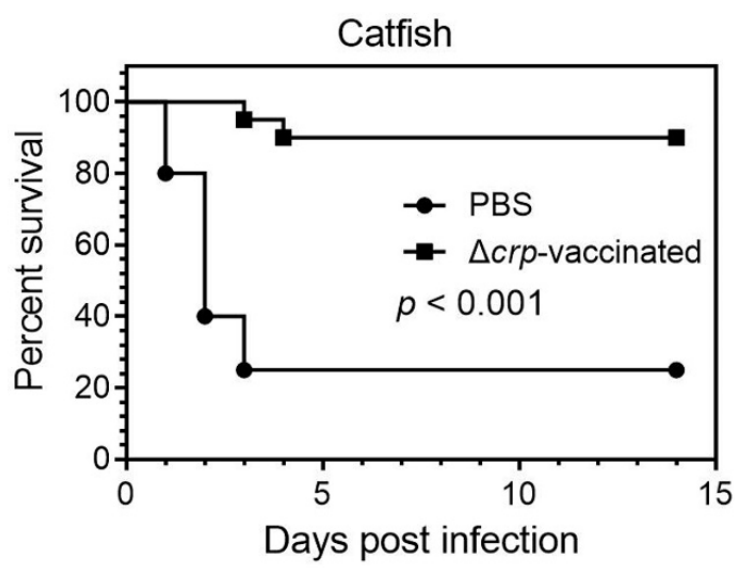

Figure 7. Immunization with $\Delta c r p$ protects zebrafish (A) and channel catfish (B) against lethal challenges with parent strain. The data shown are representative of the results of one of three independent experiments; the survival data were analyzed using the log rank (Mantel-Cox) test.

\section{Discussion}

cAMP receptor protein (CRP; also known as catabolite activator protein, CAP) is a regulatory protein in bacteria. The binding with cAMP causes a conformation change that allows CRP to bind tightly to the DNA site in the promoters of the genes it controls and interact with RNA polymerase [26,27]. Crp is widely distributed among the bacteria and relatively conserved, indicating its regulation of similar gene families related to carbohydrate metabolism [10,28], competence, growth, and virulence determinants [20,29-31].

E. piscicida is a facultative aerobic pathogen belonging to the Enterobacteriaceae family, with a wide ecological niche and host range including various species of fish [1]. In this study, a crp gene deletion mutant $(\Delta c r p)$ and a crp promoter mutant $\left(\Delta \mathrm{P}_{\text {crp }}\right)$ of E. piscicida were constructed in order to investigate the role of the crp gene in E. piscicida physiology, virulence and ability to confer immune protection to fish hosts. Replacement of the promoter of crp gene by the arabinose-regulated ara $\mathrm{C} \mathrm{P}_{\mathrm{BAD}}$ promoter, yielding $\Delta \mathrm{P}_{\text {crp }}$ in which crp transcription was arabinose dependent. In this study, $\Delta \mathrm{P}_{\text {crp }}$ was used as complement strain when the arabinose was added. Firstly, the $\Delta c r p$ mutant exhibited impaired growth in vitro. The growth delay in crp mutants seem common in diverse bacteria species, relating to its role as a global regulator [9]. Secondly, lacking of Crp, E. piscicida could not utilize maltose, which means that Crp does regulate the genes related to carbohydrate utilization and influence metabolism(s) in E. piscicida. In E. coli, the cAMP-CRP complex is required for malT expression, the transcriptional activator of five transcriptional units related to the maltose regulon [32]. The regulation mechanism between Crp and maltose operon in E. piscicida still needs further investigation. Thirdly, the $\Delta c r p$ mutant also loses motility which is conferred by flagella of bacterial pathogens [33]. Motility is critical 
for cell invasion and is associated with chemotaxis and secretion of effector proteins that act as virulence factors [34,35].

Considering this, we conducted a competitive infection assay and a fish serum suvival assay to compare systemic infection capacity of $\Delta c r p$ and the parent strains. The $\Delta c r p$ mutant showed poor colonization in different tissues and a weakened survival rate against catfish serum, indicating its attenuated evasion capacity against host immune clearance. In further virulence assessment assays, the $\mathrm{LD}_{50} \mathrm{~s}$ of the $\Delta c r p$ mutant in zebrafish and channel catfish increased by 40 -fold and 10-fold, respectively, comparing to the parent strain, and $\Delta c r p$ mutant infection group exhibited a delayed death (in the zebrafish) and mild symptoms (in the channel catfish). This was consistent with previous study that E. piscicida CK216, a $\Delta c r p$ mutant strain exhibits 1000-fold attenuation comparing to wild type strain in gold fish [36]. Indeed, recent unpublished work in our laboratory indicates that $\Delta c r p$ mutant could evoke host controllable inflammation which could effectively contribute to bacterial clearance, while J118 strain infection evoked excessive inflammatory cytokine production in the susceptible organ liver and trunk kidney, leading to severe tissue injury. Therefore, the above results support that Crp contributes to the virulence of E. piscicida and that the $\Delta c r p$ mutant is attenuated.

Being a mucosal facultative intracellular pathogen, E. piscicida is an ideal candidate to develop a live attenuated vaccine for the aquaculture industry, which could elicit both humoral and cellular immune responses [37]. We further evaluated the immune protection mediated by the $\Delta c r p$ mutant in zebrafish and catfish fingerlings. And as expected, the $\Delta c r p$ mutant group had a significantly higher survival rate than the PBS control group. We determined that the $\triangle c r p$ mutant could elicit host immune responses and offer moderate protection in zebrafish and catfish. Thence, the introduction of a $\Delta c r p$ mutation could be considered as part of genetic plan to construct an E. piscicida live attenuated vaccine in further studies.

\section{Conclusions}

In summary, we conclude that $\mathrm{Crp}$ is involved in the regulation of maltose utilization and is required for flagella synthesis in E. piscicida. More importantly, $\mathrm{Crp}$ is also required for E. piscicida resistance against host immune elimination and that it contributes to the systemic dissemination and virulence of this bacterium. Finally, the $\Delta c r p$ mutant could be a promising vaccine candidate for further genetic modification to control the E. piscicida infections.

Supplementary Materials: The NCBI accession numbers of Crps were as follows: Edwardsiella piscicida (WP_000242755.1); Edwardsiella ictaluri (WP_000242758.1); Escherichia coli (NP_417816.1); Enterobacter cloacae (WP_000242758.1); Shigella flexneri (NP_709132.1); Salmonolla Typhimurium (NP_462369.1); Salmonolla Gallinarum (WP_000242751.1); Citrobacter freundii (WP_000242758.1); Yersinia pestis (YP_002345259.1); Yersinia ruckeri (WP_004718520.1);Yersinia pseudotubercuiosis (WP_002212297.1); Aeromonas hydrophila (YP_855526.1); Aeromonas salmonicida (WP_005311762.1); Klebsiella pneeumoniae (WP_000242758.1); Shigella flexneri (NP_709132.1); Vibrio cholera (WP_000242749.1); Proteus mirabilis (WP_004246872.1); Actinobacillus pleuropneumoniae (WP_005618610.1); Pasteurella multocida (WP_005723550.1); Haemophilus influenza (NP_439118.1); Pseudomonas aerugibosa (YP_006960515.1); Xanthomonas campestris (NP_635866.1); Flavobacterium psychrophilum (YP_001295622.1); Microcystis aeruginosa (WP_002793798.1); Clostridium perfringens (WP_003455357.1); Staphylococcus aureus (WP_000138218.1); Bacillus cereus (NP_830249.1); Mycobacterium tuberculosis (NP_218193.1); Methylobacterium extorquens (WP_012255086.1).

Author Contributions: Data curation, P.Z., X.H., X.Y. and Q.X.; Funding acquisition, R.C.I. and Y.Z.; Software, F.Z. and T.Y.; Supervision, R.C.I. and Y.Z.; Writing—original draft, X.H. and Y.Z.; Writing一review \& editing, Y.-A.Z. and R.C.I. All authors have read and agreed to the published version of the manuscript.

Funding: The study was supported by the National Natural Science Foundation of China (31772889), the USDA/NIFA grant (2017-05723), the National Innovation and Entrepreneurship Training Program for College Students (201810504102), the Fundamental Research Funds for the Central Universities (2662017JC040, 2662016QD010).

Conflicts of Interest: The authors declare no conflict of interest. 


\section{References}

1. Abayneh, T.; Colquhoun, D.J.; Sorum, H. Edwardsiella piscicida sp. Nov., a novel species pathogenic to fish. J. Appl. Microbiol. 2013, 114, 644-654. [CrossRef] [PubMed]

2. Mohanty, B.R.; Sahoo, P.K. Edwardsiellosis in fish: A brief review. J. Biosci. 2007, 32, 1331-1344. [CrossRef] [PubMed]

3. Loch, T.P.; Hawke, J.P.; Reichley, S.R.; Faisal, M.; Del Piero, F.; Griffin, M.J. Outbreaks of edwardsiellosis caused by Edwardsiella piscicida and Edwardsiella tarda in farmed barramundi (Lates calcarifer). Aquaculture 2017, 481, 202-210. [CrossRef]

4. Bujan, N.; Toranzo, A.E.; Magarinos, B. Edwardsiella piscicida: A significant bacterial pathogen of cultured fish. Dis. Aquat. Organ. 2018, 131, 59-71. [CrossRef] [PubMed]

5. Ma, R.; Yang, G.; Xu, R.; Liu, X.; Zhang, Y.; Ma, Y.; Wang, Q. Pattern analysis of conditional essentiality (PACE)-based heuristic identification of an in vivo colonization determinant as a novel target for the construction of a live attenuated vaccine against Edwardsiella piscicida. Fish Shellfish Immunol. 2019, 90, 65-72. [CrossRef]

6. Wang, Q.; Yang, M.; Xiao, J.; Wu, H.; Wang, X.; Lv, Y.; Xu, L.; Zheng, H.; Wang, S.; Zhao, G.; et al. Genome sequence of the versatile fish pathogen Edwardsiella tarda provides insights into its adaptation to broad host ranges and intracellular niches. PLoS ONE 2009, 4, e7646. [CrossRef]

7. Munang'andu, H.M. Intracellular bacterial infections: A challenge for developing cellular mediated immunity vaccines for farmed fish. Microorganisms 2018, 6, E33. [CrossRef]

8. Yamasaki, M.; Araki, K.; Maruyoshi, K.; Matsumoto, M.; Nakayasu, C.; Moritomo, T.; Nakanishi, T.; Yamamoto, A. Comparative analysis of adaptive immune response after vaccine trials using live attenuated and formalin-killed cells of Edwardsiella tarda in ginbuna crucian carp (Carassius auratus langsdorfii). Fish Shellfish Immunol. 2015, 45, 437-442. [CrossRef]

9. Green, J.; Stapleton, M.R.; Smith, L.J.; Artymiuk, P.J.; Kahramanoglou, C.; Hunt, D.M.; Buxton, R.S. Cyclic-AMP and bacterial cyclic-AMP receptor proteins revisited: Adaptation for different ecological niches. Curr. Opin. Microbiol. 2014, 18, 1-7. [CrossRef]

10. Colton, D.M.; Stabb, E.V. Rethinking the roles of CRP, cAMP, and sugar-mediated global regulation in the Vibrionaceae. Curr. Genet. 2016, 62, 39-45. [CrossRef]

11. Shimada, T.; Fujita, N.; Yamamoto, K.; Ishihama, A. Novel roles of cAMP receptor protein (CRP) in regulation of transport and metabolism of carbon sources. PLoS ONE 2011, 6, e20081. [CrossRef] [PubMed]

12. Curtiss, R.; Kelly, S.M. Salmonella typhimurium deletion mutants lacking adenylate cyclase and cyclic AMP receptor protein are avirulent and immunogenic. Infect. Immun. 1987, 55, 3035-3043. Available online: https://iai.asm.org/content/55/12/3035 (accessed on 1 December 1987). [CrossRef] [PubMed]

13. Skorupski, K.; Taylor, R.K. Cyclic AMP and its receptor protein negatively regulate the coordinate expression of cholera toxin and toxin-coregulated pilus in Vibrio cholerae. Proc. Natl. Acad. Sci. USA 1997, 94, 265-270. [CrossRef] [PubMed]

14. Petersen, S.; Young, G.M. Essential role for cyclic AMP and its receptor protein in Yersinia enterocolitica virulence. Infect. Immun. 2002, 70, 3665-3672. [CrossRef] [PubMed]

15. Stapleton, M.; Haq, I.; Hunt, D.M.; Arnvig, K.B.; Artymiuk, P.J.; Buxton, R.S.; Green, J. Mycobacterium tuberculosis cAMP receptor protein (Rv3676) differs from the Escherichia coli paradigm in its cAMP binding and DNA binding properties and transcription activation properties. J. Biol. Chem. 2010, 285, 7016-7027. [CrossRef] [PubMed]

16. Kennedy, M.J.; Yancey, R.J.; Sanchez, M.S.; Rzepkowski, R.A.; Kelly, S.M.; Curtiss, R. Attenuation and immunogenicity of $\Delta$ cya $\Delta$ crp derivatives of Salmonella choleraesuis in pigs. Infect. Immun. 1999, 67, 4628-4636. Available online: http://www.ncbi.nlm.nih.gov/pubmed/10456909 (accessed on 12 June 2019). [CrossRef] [PubMed]

17. Sheoran, A.S.; Timoney, J.F.; Tinge, S.A.; Sundaram, P.; Curtiss, R. Intranasal immunogenicity of a $\Delta c y a$ $\Delta$ crp-pabA mutant of Salmonella enterica serotype Typhimurium for the horse. Vaccine 2001, 19, 3787-3795. Available online: http://www.ncbi.nlm.nih.gov/pubmed/11395214 (accessed on 12 June 2019). [CrossRef]

18. Rosu, V.; Chadfield, M.S.; Santona, A.; Christensen, J.P.; Thomsen, L.E.; Rubino, S.; Olsen, J.E. Effects of crp deletion in Salmonella enterica serotype Gallinarum. Acta Vet. Scand. 2007, 49, 14. [CrossRef] 
19. Valderrama, K.; Saravia, M.; Santander, J. Phenotype of Aeromonas salmonicida sp. salmonicida cyclic adenosine 3', 5'-monophosphate receptor protein (Crp) mutants and its virulence in rainbow trout (Oncorhynchus mykiss). J. Fish Dis. 2017, 40, 1849-1856. [CrossRef]

20. Santander, J.; Mitra, A.; Curtiss, R. Phenotype, virulence and immunogenicity of Edwardsiella ictaluri cyclic adenosine 3',5'-monophosphate receptor protein (Crp) mutants in catfish host. Fish Shellfish Immunol. 2011, 31, 1142-1153. [CrossRef]

21. Zhang, Y. I-TASSER server for protein 3D structure prediction. BMC Bioinform. 2008, 9, 40. [CrossRef] [PubMed]

22. Ji, Y.; Li, J.; Qin, Z.; Li, A.; Gu, Z.; Liu, X.; Lin, L.; Zhou, Y. Contribution of nuclease to the pathogenesis of Aeromonas hydrophila. Virulence 2015, 6, 515-522. [CrossRef] [PubMed]

23. Kong, Q.; Liu, Q.; Roland, K.L.; Curtiss, R. Regulated delayed expression of rfaH in an attenuated Salmonella enterica serovar typhimurium vaccine enhances immunogenicity of outer membrane proteins and a heterologous antigen. Infect. Immun. 2009, 77, 5572-5582. [CrossRef] [PubMed]

24. Chen, D.D.; Li, J.H.; Yao, Y.Y.; Zhang, Y.A. Aeromonas hydrophila suppresses complement pathways via degradation of complement C3 in bony fish by metalloprotease. Fish Shellfish Immunol. 2019, 94, 739-745. [CrossRef]

25. Behreans, A.L.; Karber, L. Determination of LD50. In Screening Methods in Pharmacology; Academic Press: New York, NY, USA, 1953; p. 60.

26. Kolb, A.; Busby, S.; Bue, H.; Garges, S.; Adhya, S. Transcriptional regulation by cAMP and its receptor protein. Annu. Rev. Biochem. 1993, 62, 749-795. [CrossRef]

27. Weber, I.T.; Steitz, T.A. Structure of a complex of catabolite gene activator protein and cyclic AMP refined at 2.5 Å resolution. J. Mol. Biol. 1987, 198, 311-326. [CrossRef]

28. Gorke, B.; Stulke, J. Carbon catabolite repression in bacteria: Many ways to make the most out of nutrients. Nat. Rev. Microbiol. 2008, 6, 613-624. [CrossRef]

29. Botsford, J.L.; Harman, J.G. Cyclic AMP in prokaryotes. Asm. J. 1992, 56, 100-122. Available online: https://mmbr.asm.org/content/56/1/100 (accessed on 1 March 1992). [CrossRef]

30. Busby, S.; Ebright, R.H. Transcription activation by catabolite activator protein (CAP). J. Mol. Biol. 1999, 293, 199-213. [CrossRef]

31. Chandler, M.S. The gene encoding cAMP receptor protein is required for competence development in Haemophilus influenzae Rd. Proc. Natl. Acad. Sci. USA 1992, 89, 1626-1630. [CrossRef]

32. Chagneau, C.; Heyde, M.; Alonso, S.; Portalier, R.; Laloi, P. External-pH-dependent expression of the maltose regulon and ompF gene in Escherichia coli is affected by the level of glycerol kinase. J. Bacteriol. 2001, 183, 5675-5683. [CrossRef] [PubMed]

33. Liang, W.; Pascual-Montano, A.; Silva, A.J.; Benitez, J.A. The cyclic AMP receptor protein modulates quorum sensing, motility and multiple genes that affect intestinal colonization in Vibrio cholera. Microbiology 2007, 153, 2964-2975. [CrossRef] [PubMed]

34. Barrero-Tobon, A.M.; Hendrixson, D.R. Flagellar biosynthesis exerts temporal regulation of secretion of specific Campylobacter jejuni colonization and virulence determinants. Mol. Microbiol. 2014, 93, 957-974. [CrossRef] [PubMed]

35. Josenhans, C.; Suerbaum, S. The role of motility as a virulence factor in bacteria. Int. J. Med. Microbiol. 2002, 291, 605-614. Available online: http://www.urbanfischer.de/journals/ijmm (accessed on 1 March 2002). [CrossRef]

36. Choe, Y.; Park, J.; Yu, J.E.; Oh, J.I.; Kim, S.; Kang, H.Y. Edwardsiella piscicida lacking the cyclic AMP receptor protein (Crp) is avirulent and immunogenic in fish. Fish Shellfish Immunol. 2017, 68, 243-250. [CrossRef]

37. Xiao, J.; Chen, T.; Liu, B.; Yang, W.; Wang, Q.; Qu, J.; Zhang, Y. Edwardsiella tarda mutant disrupted in type III secretion system and chorismic acid synthesis and cured of a plasmid as a live attenuated vaccine in turbot. Fish Shellfish Immunol. 2013, 35, 632-641. [CrossRef]

(C) 2020 by the authors. Licensee MDPI, Basel, Switzerland. This article is an open access article distributed under the terms and conditions of the Creative Commons Attribution (CC BY) license (http://creativecommons.org/licenses/by/4.0/). 